\title{
Treating atrial fibrillation with radiofrequency ablation to reverse changes in microRNAs regulating the ion-channel proteins
}

\author{
Yue YN, Li B, Xu GY, Yang SX \\ Department of Cardiovascular, Beijing Shijitan Hospital of China Capital Medical University, \\ Beijing, China. yangsx_ysx02@163.com
}

\begin{abstract}
OBJECTIVE: To investigate the possible molecular mechanisms of radiofrequency ablation (RFA) for treating atrial fibrillation (AF) and the microRNA (miRNA) target for intervention in the future.

METHODS: We examined the changes in miRNAs regulating the atrial ion-channel proteins across the whole genome. We compared findings from $90 \mathrm{AF}$ patients with those from 90 healthy subjects before RFA and three months after RFA.

RESULT: Twenty-one miRNAs regulating ion-channel proteins were differentially expressed more than tenfold, and the findings were completely reversed after RFA as compared with the pre-RFA results. The colonial regulating effects of miRNAs regulating the outward $\mathrm{K}^{+}$current channels such as those for the ultra-rapid delayed rectifier potassium current (Ikur), voltage-dependent delayed rectifier potassium current (Ikr), and delayed rectifier potassium channel current (Iks) were more unanimous and stronger, while this was not the case for miRNAs regulating the L-type $\mathrm{Ca}^{2+}$ current and INa current channels. Generally, miR-1266 levels were increased in the blood but down-regulated in the rheumatic atrial tissue, while a dual luciferase test indicated that SCN5A was the direct target gene of miR-1266.

CONCLUSION: Using RFA to treat AF may have an impact via reversing the changes in miRNAs regulating the ion-channel proteins, especially for outward $\mathrm{K}+$ current channels such as Ikur, Ikr, and Iks, which may play a major role in electrical remodeling in AF. It may be that miR-1266 is an antiarrhythmic miRNA and an AF intervention target in the future (Tab. 2, Fig. 4, Ref. 46). Text in PDF www.elis.sk KEY WORDS: atrial fibrillation, miRNA, genomics, radiofrequency ablation, ion-channel proteins.
\end{abstract}

\section{Introduction}

It is well known that the pathogenesis of atrial fibrillation (AF) is very complex. It involves multiple factors (genes, miRNA regulation, target changes (1-5), etc.) that result in electrical and structural remodeling, and further contribute to paroxysmal AF gradually evolving into persistent and permanent AF. Atrial fibrillation radiofrequency ablation (AF-RFA) is a primary treatment method for patients with $\mathrm{AF}$ at present $(6,7)$. However, the postoperative recurrence rate is still high (7), and the molecular mechanisms and recurrence mechanism of RFA treatments remain unclear.

Nevertheless, more attention is now being paid to the miRNA regulating mechanism in relation to the genesis and development of AF. David et al (8), for example, investigated the relationship between circulating microRNAs and AF. Based on our own AF-RFA clinical practice and previous miRNA studies (9-13), the current authors have also shown that miRNAs are involved in the

Department of Cardiovascular, Beijing Shijitan Hospital of China Capital Medical University, Beijing, China

Address for correspondence: Shuixiang Yang, MD, Department of Cardiovascular, Beijing Shijitan, Hospital of China Capital Medical University, No. 45 of Changchun Street, Xicheng District, Beijing 100053, China. Phone: +86.10 .63925588 , Fax: +86.10 .63925588$ regulation of AF and that RFA can reverse the abnormal circulation of microRNA expression in patients with AF. Recently, the miRhythm study (14) has revealed that the plasma levels of miRs -21 and -150 increase three-fold after AF ablation. However, all these miRNA changes are mainly involved in atrial structural remodeling, whereas the alteration of miRNAs regulating the ionchannel proteins has not been studied.

The findings of the present study reveal that some miRNAs regulating the ion-channel proteins are changed or reversed by RFA, especially for channels with outward $\mathrm{K}+$ currents such as the ultra-rapid delayed rectifier potassium current (Ikur), voltagedependent delayed rectifier potassium channel (Ikr), and delayed rectifier potassium channel current (Iks). This may rebalance the ion flow and reverse the electrical remodeling caused by AF. Furthermore, it is our attempt to detect which channel plays an important role in ion-channel remodeling and associated regulating miRNA changes after RFA, and to identify the miRNA's regulating mechanism and intervening targets.

\section{Materials and methods}

\section{Study population}

The 90 AF radiofrequency ablation patients chosen for this study were admitted to the researchers' hospital between January 
2013 and June 2015. The group consisted of three subgroups divided according to case type, with 30 patients in each subgroup: paroxysmal (AF persisted for $\leq 7$ days), persistent (AF persisted for $>7$ days and $<1$ year), and permanent (AF persisted for $\geq 1$ year). Each patient had undergone more than five electrocardiograms at different times to confirm their diagnosis. The exclusion criteria were as follows: patients aged $>80$ years and patients with hyperthyreosis or diabetes mellitus, poor control of blood pressure (>140 and/or $90 \mathrm{mmHg}$ ), left ventricular dysfunction ( $\mathrm{EF}<40 \%$ ), severe coronary artery disease, hepatic and/or renal dysfunction, acute and chronic infectious diseases, and myocardial structural lesions. Any patients who had been taking angiotensin-converting enzyme inhibitors, angiotensin receptor inhibitors, statin drugs to control hypertension and hyperlipemia, beta-blockers, or other anti-arrhythmic medicines to control their ventricular rate were accepted only if they had stopped taking these at least five days before operation.

All methods used in this study were in accordance with the China National Ministry of Public Health's "Biomedical research ethics review regulations," China Food and Drug Administration's "Guiding principles of drug clinical trial ethic review," and Declaration of Helsinki. The protocol of the study was approved by the Medical Ethics Committee of Shijitan Hospital. Every patient and one of their family members had to agree to participate and sign the informed consent form, and the written informed consent was obtained from all subjects before their RFA treatment.

\section{Operation procedure}

Encircling isolation of pulmonary vein ostium/orifice (EPVOI) was performed in paroxysmal AF patients, and " $2 \mathrm{O}+3 \mathrm{~L}$ " in persistent and permanent AF patients, i.e., EPVOI+3 lines: left atrial

Tab. 1. The main miRNAs regulating the ion channel proteins expressed differently before and after RFA.

\begin{tabular}{lccccc}
\hline \multicolumn{2}{c}{$\begin{array}{c}\text { pre-RFA/N.C. } \\
(\mathrm{n}=90)\end{array}$} & & & \multicolumn{2}{c}{$\begin{array}{c}\text { pre-/post-RFA } \\
(\mathrm{n}=90)\end{array}$} \\
\cline { 2 - 3 } miRNAs & Difference Fold & $\mathrm{p}$ & & Difference Fold & $\mathrm{p}$ \\
\cline { 2 - 3 } \cline { 5 - 6 } hsa-miR-1266 & $1.96 \pm 0.025$ & $<0.01$ & & $249.86 \pm 0.218$ & $<0.001$ \\
hsa-miR-377-5p & $4.27 \pm 0.039$ & $<0.01$ & & $102.90 \pm 0.237$ & $<0.001$ \\
hsa-miR-3664-5p & $-8.88 \pm 0.089$ & $<0.01$ & & $-46.06 \pm 0.156$ & $<0.001$ \\
hsa-miR-1284 & $1.94 \pm 0.056$ & $<0.01$ & & $-27.13 \pm 0.217$ & $<0.001$ \\
hsa-miR-4796-5p & $2.68 \pm 0,106$ & $<0.01$ & & $-23.62 \pm 0.089$ & $<0.001$ \\
hsa-miR-296-3p & $4.30 \pm 0.049$ & $<0.01$ & & $-10.58 \pm 0.820$ & $<0.001$ \\
hsa-miR-4666a-3p & $-3.69 \pm 0.026$ & $<0.01$ & & $13.83 \pm 0.093$ & $<0.001$ \\
hsa-miR-30d-5p & $-7.50 \pm 0.021$ & $<0.01$ & & $14.15 \pm 0.088$ & $<0.001$ \\
hsa-miR-4306 & $-8.33 \pm 0.035$ & $<0.01$ & & $20.90 \pm 0.142$ & $<0.001$ \\
hsa-miR-328 & $-1.79 \pm 0.024$ & $<0.01$ & & $28.49 \pm 0.172$ & $<0.001$ \\
hsa-miR-101-3p & $18.52 \pm 0.061$ & $<0.01$ & & $34.94 \pm 0.181$ & $<0.001$ \\
hsa-miR-146b-5p & $11.16 \pm 0.039$ & $<0.01$ & & $39.16 \pm 0.219$ & $<0.001$ \\
hsa-miR-186-5p & $12.05 \pm 0.014$ & $<0.01$ & & $101.88 \pm 0.235$ & $<0.001$ \\
hsa-miR-151a-3p & $11.83 \pm 0.016$ & $<0.01$ & & $104.79 \pm 0.213$ & $<0.001$ \\
hsa-miR-98-5p & $-7.02 \pm 0.085$ & $<0.01$ & & $130.54 \pm 0.173$ & $<0.001$ \\
hsa-miR-155-5p & $-3.17 \pm 0.027$ & $<0.01$ & & $132.57 \pm 0.218$ & $<0.001$ \\
hsa-miR-224-5p & $30.49 \pm 0.053$ & $<0.01$ & & $148.29 \pm 0.328$ & $<0.001$ \\
hsa-miR-361-5p & $-5.12 \pm 0.026$ & $<0.01$ & & $150.68 \pm 0.254$ & $<0.001$ \\
hsa-miR-152 & $16.34 \pm 0.071$ & $<0.01$ & & $184.27 \pm 0.356$ & $<0.001$ \\
hsa-miR-199a-3p & $11.30 \pm 0.062$ & $<0.01$ & & $221.98 \pm 0.562$ & $<0.001$ \\
hsa-miR-199b-3p & $11.30 \pm 0.051$ & $<0.01$ & & $221.98 \pm 0.751$ & $<0.001$ \\
\hline
\end{tabular}

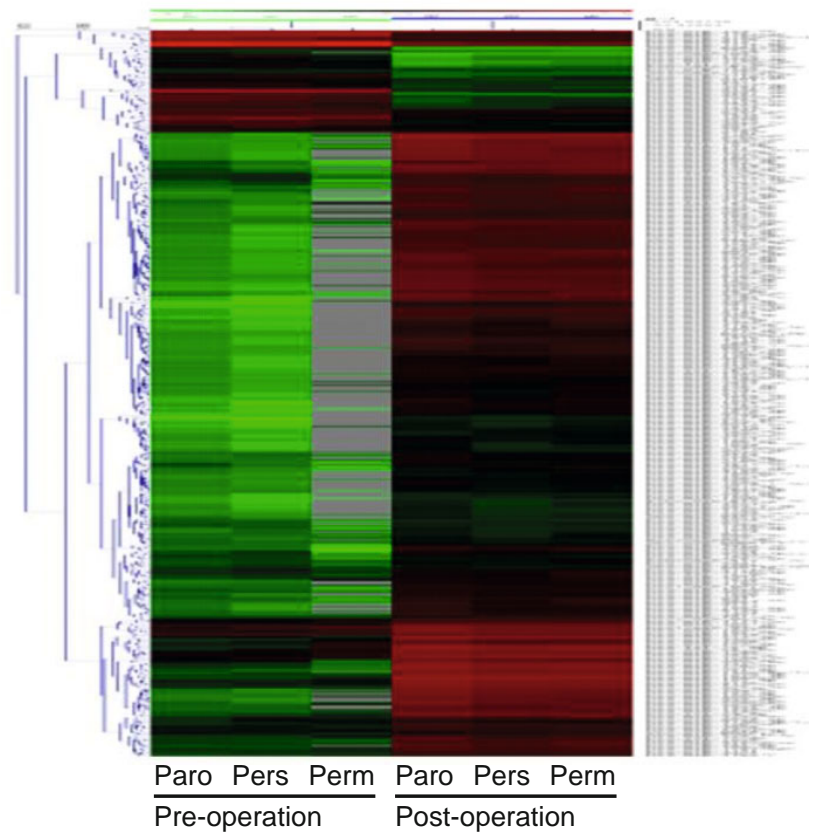

Fig. 1. Cluster analysis of miRNA expression in AF patients pre- and post-RFA (Paro = Paroxysmal AF $;$ Pers = Persistent AF ; Perm = Permanent AF). The color changes in Figure 1 show that the miRNA changes were entirely different in the three different AF groups in pre-RFA, but very similar across the same groups post-RFA; this indicates that AF-RFA reversed or changed the miRNA abnormalities in AF patients.

roof line and lines at the narrow parts of annuli of mitral and tricuspid valves. The patients with no recurrence 3 months after their AF ablation operation were placed into the subgroup (three Holter monitor tests done in one-month intervals after the operation showed no AF recurrence).

\section{Plasma collection and storage}

Peripheral blood (PB) samples were taken from patients before RFA and at 3 months after RFA. In each instance, a whole-blood sample $(4 \mathrm{ml})$ was drawn into EDTA-containing tubes and separated into plasma and cellular fractions by centrifugation at $1,500 \mathrm{~g}$ for 15 minutes. The supernatant was then transferred to RNase/DNasefree tubes and stored at $-80^{\circ} \mathrm{C}$.

\section{RNA isolation}

Total plasma RNA was harvested using the TRIzol reagent (Invitrogen Life Technology) and miRNeasy mini kit (Qiagen) according to the manufacturers' instructions. In summary, $250 \mu \mathrm{l}$ of EDTA-containing plasma was transferred to an Eppendorf tube and mixed thoroughly with TRIzol reagent, incubated for 5 minutes at room 
Tab. 2. Results of miRNA target prediction.

\begin{tabular}{ll}
\hline miRNA & Target genes of ion channel proteins \\
\hline hsa-miR-1266 & SCN5A, KCNH2, KCNE1, KCNA6, KCNA7, KCNC3, KCNB1, KCNG4, \\
& KCNH5, KCNH8, KCNP4, KCNJ1, KCNJ11, KCNJ3, KCNJ5, KCNK1, \\
\hline hsa-miR-377-5p & GJA5, KCNQ1, CACNA1C \\
\hline hsa-miR-3664-5p & KCNC1, CACNA1B, CAMTA2 \\
\hline hsa-miR-1284 & HCN4, SCN5A, KCNC1 \\
\hline hsa-miR-4796-5p & HCN4 \\
\hline hsa-miR-296-3p & KCNQ1, HCN1, GJA5, KCNH2 \\
\hline hsa-miR-4666a-3p & KCNE4, KCNQ3, KCNC1, KCNG3, KCNJ3, HCN4, CACNA1C, CAM- \\
\hline hsa-miR-30d-5p & KAP1, CAMTA1 \\
\hline hsa-miR-4306 & KCNC4, HCN4, CACNA1C, KCNN2, KCNN3, KCNC1, KCNC4 \\
\hline hsa-miR-328 & CACNA1C, CACNA1B \\
\hline hsa-miR-101-3p & KCNH2, GJA1, KCNE1, KCNA5 \\
\hline hsa-miR-146b-5p & HCN1, SCN5A \\
\hline hsa-miR-186-5p & CACNA1C, KCNJ2, KCNC4, KCNA5 \\
\hline hsa-miR-151a-3p & KCNA5, HCN1 \\
\hline hsa-miR-98-5p & KCNC4, KCNJ2, SCN5A \\
\hline hsa-miR-155-5p & KCNA5, KCNN3, HCN3, KCNH2, KCNN2, KCNC1, GJA5, \\
\hline hsa-miR-224-5p & KCND2, KCNN2, GJA3 \\
\hline hsa-miR-361-5p & KCNQ5, KCNJ2, RYR2, KCNA5, CaMK2D \\
\hline hsa-miR-152 & KCNH2, KCNN3 \\
\hline hsa-miR-199a/b-3p & KCNH2, KCNN2, KCND2, GJA3 \\
\hline
\end{tabular}

dard protocol for the ABI PRISM 7000HT Sequence Detection System was as follows: $10 \mathrm{~s}$ at $95{ }^{\circ} \mathrm{C}$ and $1 \mathrm{~min}$ at $60{ }^{\circ} \mathrm{C}$ for 40 cycles. The comparison of miRNA expression can be quantified using the following formula: $2^{-\Delta \Delta C T}$.

\section{MicroRNA target prediction}

MicroRNA targets were predicted using the target-prediction programs miRanda, TargetScan, and miRbase. The Database for Annotation, Visualization, and Integrated Discovery was used to identify the pathway distribution of predicted targets. Pathways were presented according to the Kyoto Encyclopedia of Genes and Genomes (KEGG) database, which is a database of biological systems that contains information on the genetic building blocks of genes and proteins.

\section{Luciferase combination experiment}

After the miR-1266 plasmid (pcDNA 6.2-GW/miRNA-1266) and negative control plasmid (pcDNA6.2-GW/miRNA NC) had been covalently transfected with the SCN5A recombinant luciferase plasmid temperature, and subsequently mixed with $140 \mu \mathrm{l}$ chloroform. The aqueous phase containing the RNA was carefully removed, and the RNA was precipitated by adding $100 \%$ ethanol. The mixture was applied to an RNeasy mini spin column and washed several times, and the RNA was eluted by adding $25 \mu \mathrm{l}$ of RNase-free water. The RNA was then stored at $-80{ }^{\circ} \mathrm{C}$ until further processing.

\section{MicroRNA array and data analysis}

The RNA samples were labeled with the Exiqon miRCURY Hy3/Hy5 power labeling kit (Cat \#208032-A Exiqon) and hybridized on the miRCURY LNA Array station (version 18.0, Exqion). Scanning was performed using the Axon GenePix 4000B microarray scanner. GenePix pro V6.0 was used to read image intensity. This test was completed by Shanghai Kangcheng Biological Co., Ltd.

\section{Real-time polymerase chain reaction}

To confirm the findings obtained by analyzing the miRNA profiles, we measured dysregulated miRNA expression using realtime polymerase chain reaction (PCR). All primers used in this study were synthesized by Bioligo Technologies Ltd. (Shanghai, China). RNA was extracted from all serum samples, and from discarded valves and atrial tissues from patients with rheumatic AF who were undergoing mitral valve replacement surgery (control group: non-AF patients), to measure concentration and quality. The extraction was conducted in accordance with the manufacturer's protocols (Invitrogen Life Technology). A cDNA synthesis kit (Exiqon) was used to generate cDNA for microRNA profiling, again in accordance with the manufacturer's protocol. The stan-
(pmirGLO-SCN5A3'-untranslated region (UTR)), compared with miR-1266 plasmid and blank group, the relative luciferase activity of each group was analyzed.

\section{Statistical analysis}

The threshold value for significance that was used to define miRNA up-regulation or down-regulation was a change $>1.5$-fold, with a $\mathrm{p}<0.05$ that was calculated using a $t$-test.

\section{Results}

MicroRNA differential expression in atrial fibrillation patients before RFA and 3 months after RFA

The average age of the patients was $72.17 \pm 4.76$ years. Of the sample of patients with AF, 44 cases were female and 46 cases were male. Ninety healthy physical examination subjects, with an average age of $69.40 \pm 5.86$ years, were selected as controls.

In the group of AF patients, 503 miRNAs were up-regulated, but 81 miRNAs were down-regulated more than 1.5-fold in the post-RFA results as compared with the pre-RFA findings ( $p<$ 0.01 ). Meanwhile, the expression of 21 miRNAs that regulate key ion-channel proteins was changed more than 10-fold in post-RFA results as compared with pre-RFA findings (Tab. 1). Among these, miR-1266, miR-377-5p, miR-1284, miR-4796-5p, and miR-296$3 p$ were up-regulated before RFA more than 1.5 -fold as compared with the control group, but they were down-regulated more than 10 -fold after RFA as compared with the pre-RFA findings in the AF patients. Meanwhile, miR-101-3p, miR-146b-5p, miR-1865p, miR-151a-3p, miR-98-5p, miR-155-5p, miR-224-5p, miR- 
A

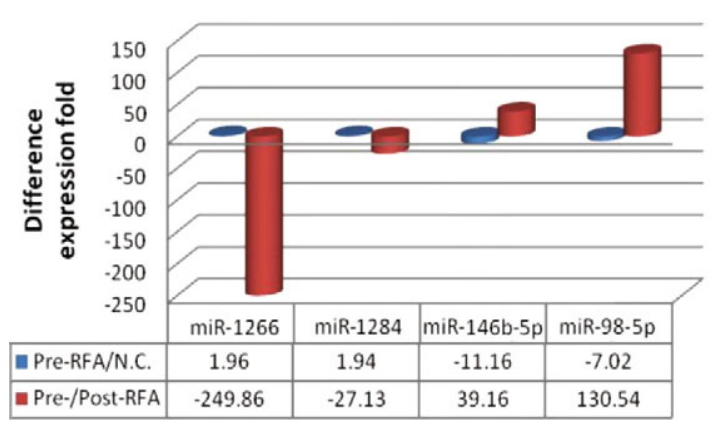

(C)

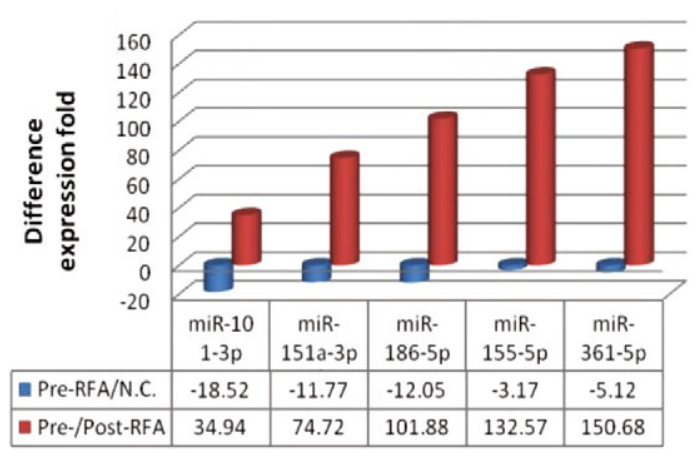

(B) miRNAs regulated by CACNA1C

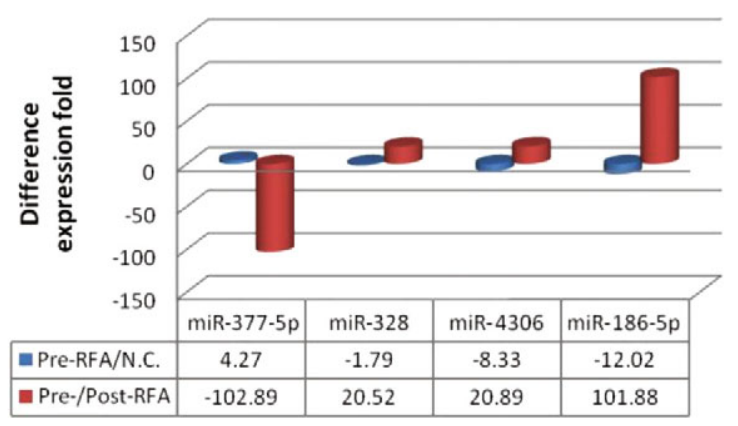

(D) miRNAs regulated by $\mathrm{KCNH} 2$

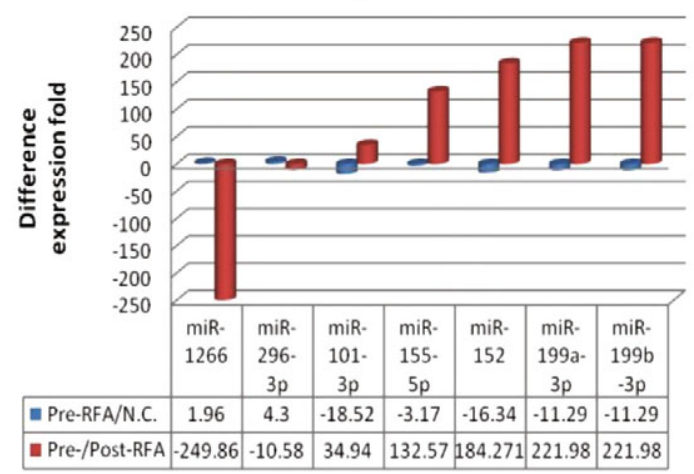

Fig. 2. The miRNAs regulated by (A) SCN5A, (B) CACNA1C, (C) KCNA5, and (D) KCNH2.

152, miR-199a-3p, and miR-199b-3p were down-regulated more than 10-fold before RFA and up-regulated more than 30-fold after RFA (Fig. 1, Tab. 1).

MicroRNA target prediction and microRNA expressions regulated by ion-channel proteins

MicroRNA targets were predicted using the target-prediction programs in miRanda, TargetScan, and miRbase. The target genes were contained in at least two databases (Tab. 2).

Based on the cardiac action potential curve and target gene prediction, several miRNAs regulated the expression of the SCN5Aencoded $\alpha$-subunit of the sodium channel, which determines the 0 phase of action potential duration (APD). These miRNAs were miR-1266, miR-1284, miR-146b-5p, and miR-98-5p (Fig. 2A).

There were four miRNAs that regulated the expression of CACNA1C, which encodes the main ion-channel protein regulating the APD plateau. These miRNAs, namely miR-377-5p, miR-328, miR-4306, and miR-186-5p were significantly altered in both pre-RFA/NC and pre-/post-RFA comparisons (Fig. 2B).

The outward potassium ion current is the major current in the action potential plateau. Five main miRNAs regulated KCNA5, which encodes the ultra-rapid delayed rectifier potassium current (Ikur) ion-channel protein. Significant changes were observed in miR-101-3p, miR-151a-3p, miR-186-5p, miR-155-5p, and miR- 361-5p, namely in both pre-RFA/NC and pre-/post-RFA comparisons (Fig. 2C).

$\mathrm{KCNH} 2$ encodes the fast-rapid delayed rectifier potassium current (Ikr) ion-channel protein, which is one of the major cardiac voltage-gated potassium channels. $\mathrm{KCNH} 2$ thus plays an important role in the action potential repolarization in relation to several miRNAs, including miR-1266, miRNA-296-3p, miR-152, miR199a-3p, and miR-199b-3p, which showed significant differences in both pre-RFA/NC and pre-/post-RFA comparisons (Fig. 2D).

$\mathrm{KCNE} 1$ encodes the subunit of the slow-rapid Iks ion-channel protein regulated by miRNAs including miR-1266, miR-4480, miR-4764-5p, and miR-101-3p, which all showed significant differences between the pre-RFA/NC and pre-/post-RFA results (Fig. 3A).

$\mathrm{KCNJ} 2$ encodes the $\alpha$-subunit of the inward rectifier potassium current (IK1), which plays an important role during repolarization. It is regulated by several miRNAs, including miR-1865p, miR-98-5p, and miR-101-3p, which significantly changed when comparing the pre-RFA/NC and pre-/post-RFA conditions (Fig. 3B).

$\mathrm{KCNC} 4$ encodes a subunit of the acetylcholine-activated $\mathrm{K}+$ current (IKAch) ion-channel protein that plays an important role in repolarization. It is regulated by several miRNAs, including miR-4306, miR-186-5p, and miR-98-5p, which were significantly 
(A) miRNAs regulated by KCNE1

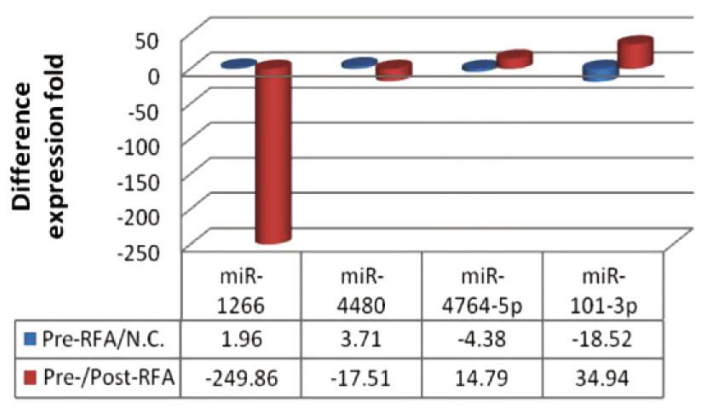

C

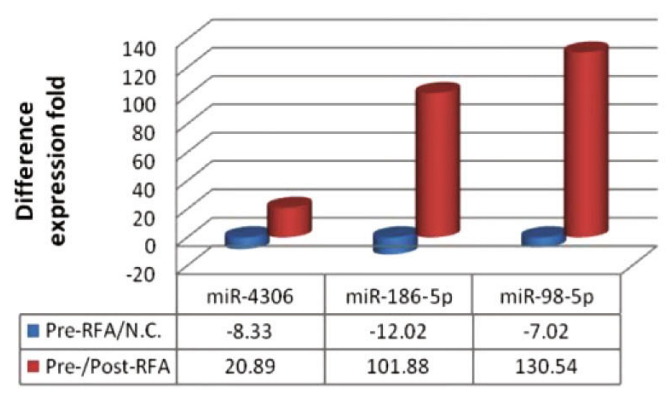

(B)

miRNAs regulated by KCNJ2

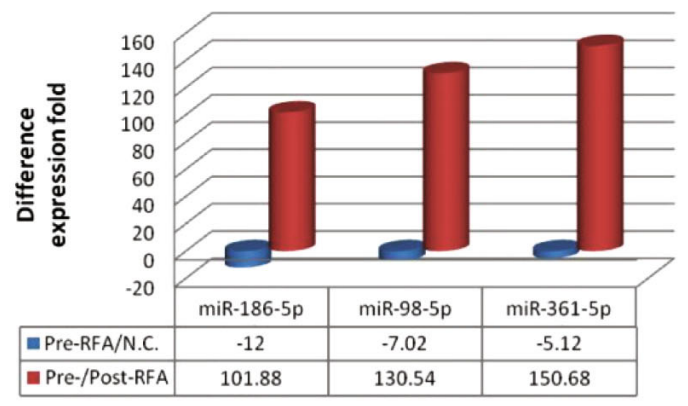

(D) miRNAs regulated by KCNN3

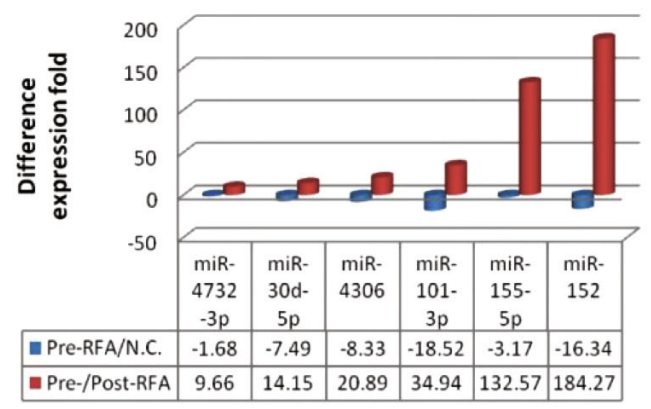

Fig. 3. The miRNAs regulated by (A) KCNE1, (B) KCNJ2, (C) KCNC4, and (D) KCNN3.

altered when comparing the pre-RFA/NC and pre-/post-RFA conditions (Fig. 3C). MiR-133b regulates KCND3, the gene encoding the transient outward $\mathrm{K}^{+}$current (Ito) ion-channel protein subunit. This miRNA was shown to increase 2.89-fold in AF patients as compared with controls pre-RFA, while it displayed a 1.98 -fold change when comparing the pre- and post-RFA conditions.

KCNN3 encodes a small-conductance calcium-activated potassium channel 3 (SK3), and it is regulated by several miRNAs, including miRNA-4732-3p, miR-30d-5p, miR-4306, miR-101-3p, miR-155-5p, and miR-152. These were all significantly altered when comparing the pre-RFA/NC and pre-/post-RFA conditions (Fig. 3D).

MicroRNA chip results and miR-1266 expression in rheumatic left atrial tissues

The chip data of the aforementioned main ion-channel-regulating microRNAs (Tab. 1) were validated using real-time PCR. The expression levels of these 21 miRNAs were confirmed against the previously observed significant up-regulation or down-regulation patterns (Fig. 4A).

The miR-1266 expression levels in the left atrial tissues of rheumatic AF patients were validated using real-time PCR. The discarded valves and atrial tissues from 20 rheumatic AF patients who were undergoing mitral valve replacement surgery were tested (control group: 20 non-AF patients undergoing the same surgery), and the findings showed that miR-1266 expression was markedly lower in the atrial tissues of the AF patients as compared with the non-AF patients $(\mathrm{p}=0.002)$ (Fig. 4B).

The luciferase relative activity was significantly lower in the miR-1266 group than in the negative control group $(p=0.002)$. Meanwhile, it was significantly higher in the blank control group as compared with both the miR-1266 and negative groups ( $\mathrm{p}<$ 0.01 ) (Fig. 4C). Therefore, the SCN5A gene was identified as a target of miR-1266.

\section{Discussion}

MicroRNAs are the key molecules that regulate cardiac ionchannel proteins. They regulate and maintain cardiac electrophysiological function by binding to the gene 3'-UTR. Abnormal regulation and expression of ion-channel proteins and the resulting changes in ion flow lead to atrial electrical remodeling. Arrhythmia is caused by an imbalance of the ion flow, which depends on the regulation of the ion-channel balance by antiarrhythmic and arrhythmic miRNAs $(13,15)$. The current research mainly investigated the effects of RFA, its possible regulating mechanisms, and its impact on the expression of miRNAs that may play an important regulatory role in ion-channel protein expression. We determined that changes in miRNA levels in AF patients may be one of the causes of the atrial electrical remodeling of AF, and 
furthermore, we observed that these changes can be significantly reversed by RFA.

\section{Sodium current}

SCN5A encodes the $\alpha$-subunit of the cardiac $\mathrm{Na}^{+}$channel and regulates sodium ion flow. The sodium channel of voltage dependence determines the effective refractory period of action potential (AERP) (16). The miRNAs related to SCN5A are shown in Figure 2A. The miR-1266 increased 1.96-fold pre-operatively, which is consistent with the RT-PCR results in the human rheumatic atrial tissue (released into the blood as shown in Fig. 4B), and decreased 249.86-fold post-operatively in AF patients. The miR-1266 and SCN5A dual luciferase experimental results indicated that SCN5A was the direct target gene of miR-1266 (Fig. 4C). SCN5A expression was down-regulated before RFA, while resulting in the reduction in sodium current density, but this was reversed dramatically after RFA. These findings are consistent with the results of animal experiments in dogs, where the atrial sodium current decreased by $28 \%$ and $52 \%$ after pacing for 7 and 42 days, respectively, while the conduction velocity was also significantly decreased (17). It has recently been shown that the $\mathrm{I}_{\mathrm{Na}}$ peak current density decreases with a decline in the pore protein Nav1.5 (18-20). However, miR-146-5p and miR-98-5p were significantly decreased (11.16-fold and 7.02-fold, respectively) before RFA, which may be the arrhythmic miRNAs, but miR-1266 and miR-1284 the antiarrhythmic miRNA; therefore, in our study, the colonial regulating effect might have been in a minor increase in INa-channel protein expression, which may be a compensatory change in response to INa current decline. Consequently, we suspect that the INa channel and current remodeling are adaptive changes following the remodeling of other ion flows $\left(\mathrm{Ca}^{2+}\right.$ and $\mathrm{K}^{+}$ current, etc.) rather than a dominant alteration.
A

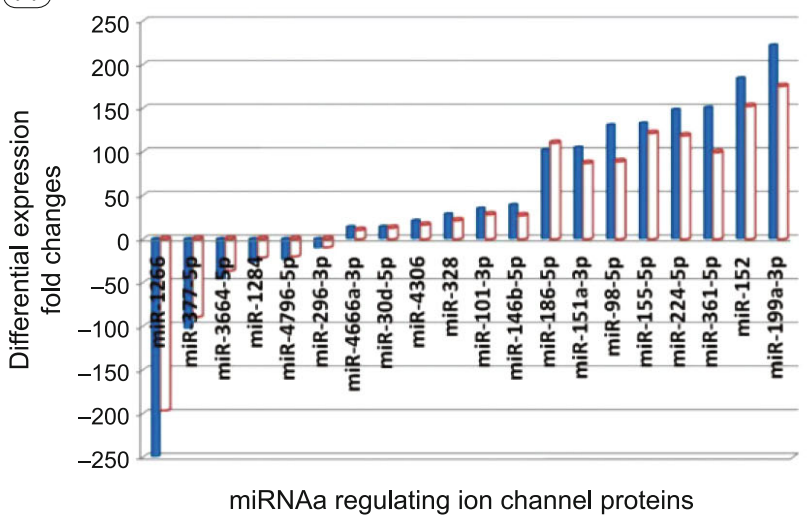

B

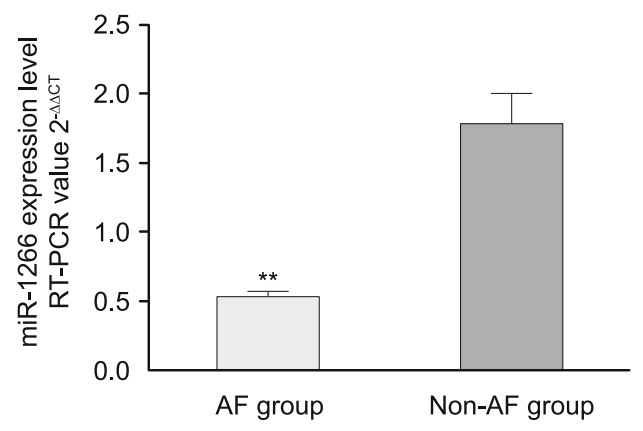

a Chip results $\quad \square \mathrm{PCR}$ results

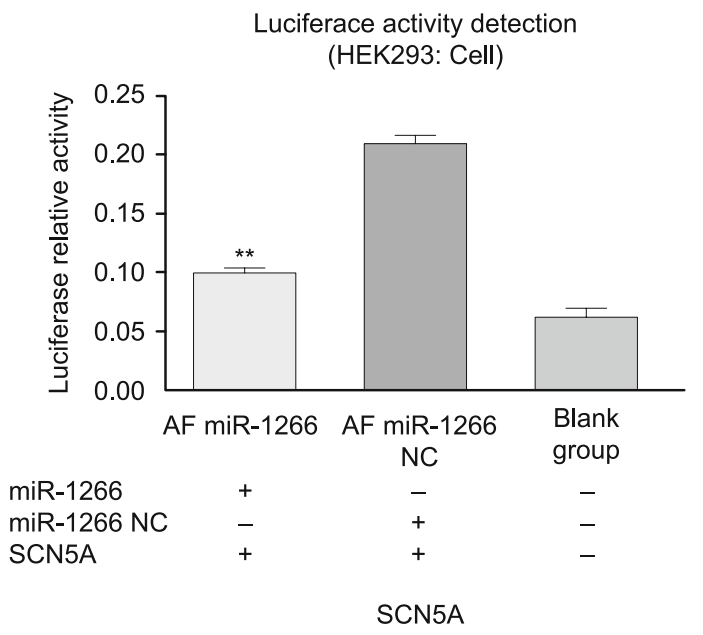

Fig. 4. miRNA chip results and miR-1266 expression in human rheumatic left atrial tissues. (A) RT-PCR verified the miRNA chip results for AF patients, comparing for pre- and post-RFA. The expression levels of these 21 miRNAs confirmed the previously observed significant upregulation or down-regulation. (B) miR-1266 expression levels in human rheumatic left atrial tissue, confirmed with RT-PCR to be markedly decreased in the atrial tissues of the AF patients compared with the non-AF mitral-valve replacement patients. (C) Combined experimental results for miR-1266 with SCN5A gene dual luciferase, indicating that the SCN5A gene is a target of miR-1266. 


\section{6-404}

\section{Calcium current}

The L-type $\mathrm{Ca}^{2+}$ channel $\alpha$-subunit gene CACNA1C was found to be regulated by several different miRNAs (Fig. 2B). The expression of miR-377-5p increased 4.27-fold before RFA and decreased 102.89-fold after RFA in AF patients. This miRNA plays a key role in the regulation of CACNA1C expression and results in a reduction in the L-type $\mathrm{Ca}^{2+}$ current $\left(\mathrm{I}_{\mathrm{CaL}}\right.$ ), shortening of APD and AERP and, in turn, in an increase in cardiac vulnerability to AF. L-type $\mathrm{Ca}^{2+}$ current reduced in about $60-70 \%$ of patients with AF. These results are consistent with other findings that have shown a decrease in $\mathrm{I}_{\mathrm{CaL}}$ in dogs undergoing rapid atrial pacing (17). Lu et al reported that miR-328 levels were elevated 3.5-fold in AF patients with a rheumatic heart valvular disease $(21,22)$, but in our study, miR-328 expression decreased 1.79-fold before RFA and increased 20.52-fold after RFA. The reason for this difference may be that our research subjects and sample differ from those in Lu et al's study. We used the blood of AF patients with no valve disease, whereas $\mathrm{Lu}$ et al took atrial tissues from AF patients who had undergone mitral-valve replacement. Therefore, the miR-328 retention in the atrial tissue may be easily induced in cases of AF susceptibility and, when released into the blood, it may inhibit AF from coming up. Our study also revealed that miR-328 and miRNAs regulating the $\mathrm{Ca}^{2+}$ channel were entirely reversed by the RFA intervention. Furthermore, our findings indicate that miR-328 is a potentially useful target site in the regulation of $\mathrm{I}_{\mathrm{CaL}}$. The levels of expression of mir-328, miR-4306, and miR-186-5p decreased before RFA, which may be for reasons similar to the INa current in terms of compensatory changes resulting from the $\mathrm{I}_{\mathrm{CaL}}$ decline and increase in the outward $\mathrm{K}^{+}$ion current.

\section{Kv1.5}

The Ikur is thought to play a major role in the repolarization process of human atrial myocytes. Ikur is carried by functional channels that are assembled using KCNA5-encoded pore-forming $\alpha$-subunits (23). The main miRNAs by which KCNA5 was observed to be regulated are shown in Figure $2 \mathrm{C}$. The regulating trend and amplitude of all miRNAs were more unanimous and larger; namely, the expression of all regulating miRNAs was deeply decreased before RFA, but augmented dramatically after RFA. This resulted in a marked increase in the KCNA5 expression before RFA and increase in outward $\mathrm{K}^{+}$current. In turn, this led to the shortening of APD and AERP, and enhanced AF susceptibility before RFA; however, these were reversed after RFA. These results are consistent with the theory of atrial remodeling in AF (24). Therefore, we believe that the Ikur current changes may play a leading role in the electrical remodeling in AF, while serving as the initiator of ion-flow changes. This view is consistent with the results regarding the effectiveness of the Ikur current inhibitor, vernakalant, in the treatment of AF (25-27). Additionally, our results are consistent with the Christophersen's study, where six novel nonsynonymous mutations were found in KCNA5 and KCNAB2 in 307 patients diagnosed with $\mathrm{AF}$, of which three mutant proteins decreased and weakened the KV1.5 current, while the other three preserved and strengthened the $\mathrm{K}^{+}$current (28), while all of them enhanced AF susceptibility.
However, another previous study (29) found out that the density of Ikur was reduced in atrial myocytes from patients with AF. In the left and right auricles of patients with chronic AF, the Ikur density was reduced by $57 \%$ and $51 \%$, respectively, which accompanied a decrease in KCNA5 expression. One possible explanation is that electrical remodeling causes an increase in KV1.5 in early $\mathrm{AF}$, but then a gradual decrease in persistent or chronic AF takes place due to the reduction in other ion currents such as $\mathrm{I}_{\mathrm{CaL}}$, etc.

\section{IKr and IKs}

$\mathrm{KCNH} 2$ encodes the $\alpha$-subunit of the voltage-dependent delayed rectifier potassium channel ( $\mathrm{Ikr}$ ), which is regulated by several kinds of miRNAs (Fig. 2D). The expression of miR-1266 increased 1.96-fold before RFA and decreased 249.86-fold after RFA, while the other associated miRNAs decreased before and increased after RFA. The synthetical levels of $\mathrm{KCNH} 2$ regulation and expression were increased, Ikr was increased, APD and ERP were shortened, and AF susceptibility was enhanced in the preRFA stage, but these were reversed in the post-RFA condition. This is consistent with the theory of electrical remodeling (30), and it has further confirmed the effectiveness of the RFA intervention.

KCNE1 is a slow-activating component of the IKs, which plays an important role in atrial repolarization, and it is regulated mainly by the miRNAs shown in Figure 3A. The expressions of miR-4480, miR4764-5p, and miR-101-3p were decreased before RFA and increased after RFA, but the miR-1266 expression showed an opposite trend. The KCNE1 synthetical expression was up-regulated, Iks was increased, APD and ERP were shortened, and AF susceptibility was enhanced in the pre-RFA stage (31), but this was reversed in the post-RFA condition. The post-RFA decrease in miR-1266 may have been correlated with ADP and relative prolongation of AERP after the treatment.

\section{IK1}

IK1, which plays an important role at the end stage of repolarization (32), was increased in patients with AF. KCNJ2 encodes the $\alpha$-subunit of the IK1 channel, which is mainly regulated by miRNAs shown in Figure 3B. Among these miRNAs, miR1865p, miR-98-5p, and miR-361-5p were down-regulated before RFA and up-regulated after RFA. This resulted in an increase in KCNJ2 levels, an enhancement in IKl, and higher AF susceptibility. However, these changes were reversed by RFA. Previous research has shown that IK1 current amplitudes and densities are increased in patients or experimental models of $\operatorname{AF}(33,34)$. Specifically in cases of chronic AF, the IK1 current density was two times higher than in the control group, which contributed to the decrease in resting membrane potential and atrial APD shortening, while the easily developed fibrillation waves triggered AF.

Ito

The findings of a previous study showed that the Ito current was reduced in human and rabbit atrial cells from patients/animals with AF (35). KCND3 encodes the pore-forming subunit KV4.3 and plays an important role in the Ito current. In the current study, miR-133, which regulates KCND3, increased before and decreased 
after RFA. The Ito reduction slowed down the phase 1 of AP and elevated the plateau, thus prolonging the ERP. The decrease in Ito was more pronounced in the left atrium than in the right atrium. This may be the basis of AF development (36-39).

\section{IKAch}

The acetylcholine-activated $\mathrm{K}^{+}$current (IKAch) is a small inward-rectifying $\mathrm{K}^{+}$current that mainly exists in the atrial muscle cell membrane (40). KCNC4 production, which is regulated by several miRNAs (Fig. 3C), plays an important role in the IKAch current. In our study, the expressions of miR-4306, miR-186-5p, and miR-98-5p were decreased before RFA and increased after RFA. This resulted in KCNC4 expression being up-regulated, IKAch being enhanced, and APD being shortened. However, a previous study (41) reported that in patients with persistent AF, the IKAch current density decreased by about $50 \%$, along with a decrease in the regulating miRNA level. A decrease in the IKAch density may result in an increase in the atrial muscle excited heterogeneity, which may promote re-entrance and trigger the occurrence $(38,42)$. This may be related with the differences between phases of AF.

SK

Recent studies have shown that SK channels are small-conductance, calcium-activated potassium channels in human atrial tissue that play an important role in the APD, thus contributing to the cardiac repolarization current (43). In the current study, KCNN3, which encodes SK3, was observed to be regulated by several miRNAs (Fig. 3D). The levels of all these miRNAs were decreased before RFA and increased after RFA, while leading to an increase in KCNN3 before RFA, enhancement of the outward potassium currents, and elevated AF susceptibility. However, other studies have shown a significant increase in miR-499 in patients with AF, thus leading to SK3 down-regulation and possibly contributing to the electrical remodeling in $\mathrm{AF}(44,45)$. These findings differ from our results; in the present study, there was no significant change in miR-499 expression, but the SK current was still increased in line with the theory of AF electrical remodeling (24). Additionally, a genome-wide association study has recently associated an intronic single-nucleotide polymorphism in KCNN3 with AF (46).

\section{Conclusion}

In conclusion, the findings of this study indicate that AF-RFA not only has the effect of electrical isolation of the pulmonary vein, but can also change or restore the main abnormalities in miRNAs regulating ion-channel proteins. This may be important for rebalancing the ion flow, reversing the electrical remodeling caused by AF, and maintaining the sinus rhythm. The miR-1266 was observed to regulate multiple ion channels, while its regulating trend was consistent with the theory of AF electoral remodeling, and its amplitude was large; therefore, it may be a suitable future target for AF intervention. However, further research with larger sample populations is still needed.

\section{References}

1. Tao H, Shi KH, Yang JJ, Li J. Epigenetic mechanisms in atrial fibrillation: New insights and future directions. Review Article. Trends Cardiovasc Med 2016; 26 (4): 306-318.

2. Yang KC, Nerbonne JM. Mechanisms contributing to myocardial potassium channel diversity, regulation and remodeling. Trends Cardiovasc Med 2016; 26 (3): 209-218.

3. Algalarrondo V, Nattel S. Potassium Channel Remodeling in Heart Disease. Cardiac Electrophysiol Clin 2016; 8 (2): 337-347.

4. Bayer JD, Roney CH, Pashaei A, Jais P, Vigmond EJ. Novel Radiofrequency Ablation Strategies for Terminating Atrial Fibrillation in the Left Atrium: A Simulation Study. Front Physiol 2016; 7: 108. Published online 2016 April 12. DOI: 10.3389/fphys.2016.00108.

5. Seitz J, Bars C, Théodore G, Beurtheret S, Lellouche N, Bremondy M et al. AF Ablation Guided by Spatiotemporal Electrogram Dispersion Without Pulmonary Vein Isolation: A Wholly Patient-Tailored Approach. JACC 2017; 69 (3): 303-321.

6. Zaman JAB, Baykaner T, Clopton P, Swarup V, Kowal RC, Daubert JD et al. Recurrent Post-Ablation Paroxysmal Atrial Fibrillation Shares Substrates With Persistent Atrial Fibrillation: An 11-Center Study. JACC: Clinical Electrophysiology (in Press).

7. Phillips KP, Walker DT. Long term outcomes from catheter ablation of very longstanding persistent atrial fibrillation. Internat J Cardiol 2017; 228: 865-869.

8. McManus DD, Lin HH, Tanriverdi K, Quercio M, Yin XY, Larson MG et al. Relations between circulating microRNAs and atrial fibrillation: data from the Framingham Offspring Study. Heart Rhythm 2014; 11 (4): 663-669. DOI: 10.1016/j.hrthm.2014.01.018.

9. Nan J, Yang SX. Effects of radiofrequency ablation on TBX5, NKx2.5 and their regulating miRNA in atrial fibrillation patients. Chin J Geria Heart Bra Ves Dis 2015; 17 (12): 1236-1239. DOI: 10.3969/j.issn.10090126.2015.12.002.

10. Li L, Su Y, Yang SX. Role of atrial fibrillation-associated miR-4279 in regulation of calcium channel protein. Chin J Geria Heart Bra Ves Dis 2015; 17 (12): 1257-1260. DOI: 10.3969/j.issn.1009-0126.2015.12.

11. Su Y, Li L, Yue YN, Yang SX. Role of atrial fibrillation-associated miR-377 in regulation of sodium ion channel protein. Chin J Geria Heart Bra Ves Dis 2015; 17 (5): 511-514. DOI: 10.3969/j.issn.10090126.2015.05.020.

12. Xu GY, Cui YX, Yue YN, Yang SX. The values of coronary circulating miRNAs in patients with atrial fibrillation. PLoS ONE 2016; 11 (11): 1-13. e0166235. DOI: 10.1371/journal.pone.0166235.

13. Cui Y, Su Y, Li L, Zhao S, Nan J, Yue Y, Yang S. (Radiofrequency ablation can reverse the abnormal circulating microRNA expression changes in patients with atrial fibrillation). Zhonghua Xin Xue Guan Bing Za Zhi 2015; 43 (12): 1051-1056.

14. McManus DD, Tanriverdi K, Lin HH, Esa N, Kinno M, Mandapati $\mathbf{D}$ et al. Plasma microRNAs are associated with atrial fibrillation and change after catheter-ablation (the miRhythm Study). Heart Rhythm 2015; 12 (1): 3-10. DOI: 10.1016/j.hrthm.2014.09.050.

15. Luo X, Yang B, Nattel S. MicroRNAs and atrial fibrillation: mechanisms and translational potential. Nature Rev Cardiol 2014; 12 (2): 80-90.

16. Zaklyazminskaya E, Dzemeshkevich $S$. The role of mutations in the SCN5A gene in cardiomyopathies. Biochim Biophys Acta - Mol Cell Res 2016; 1863 (7): 1799-1805. 
17. Gaspo R, Bosch RF, Talajic M, Nattel S. Functional mechanisms underlying tachycardia induced sustained atrial fibrillation in a chronic dog model. Circulation 1997; 96 (11): 4027-4035.

18. Kyle JW, Makielski JC. Diseases caused by mutations in Nav1.5 interacting proteins. Cardiac Electrophysiol Clin 2014; (6): 797-809.

19. Nattel S. New ideas about atrial fibrillation 50 years. Nature2002; 415: 219.

20. Christ T, Boknik P, Wöhrl S, Wettwer E, Graf EM, Bosch RF et al. L-type Ca2+ current downregulation in chronic human atrial fibrillation is associated with increased activity of protein phosphatases. Circulation 2004; 110: 2651-2657.

21. Lu Y, Zhang Y, Wang N, Pan Z, Gao X, Zhang F et al. MicroRNA-328 contributes to adverse electrical remodeling in atrial fibrillation. Circulation 2010; 122 (23): 2378-2387.

22. Soeki T, Matsuura T, Bando S, Tobiume T, Uematsu E, Ise T et al. Relationship between local production of miRNA-328 and atrial substrate remodeling in atrial fibrillation. J Cardiol (in Press).

23. Van Wagoner DR. Pharmacologic relevance of $\mathrm{K}+$ channel remodeling in atrial fibrillation. J Mol Cell Cardiol 2000; 32: 1763-1766.

24. Gaborit N, Steenman M, Lamirault G, Le Meur N, Le Bouter S, Lande $\mathbf{G}$ et al. Human atrial ion channel and transporter subunit geneexpression remodeling associated with valvular heart disease and atrial fibrillation. Circulation 2005; 112: 471-481.

25. Voigt N, Dobrev D. Atrial-Selective Potassium Channel Blockers. Review Article. Cardiac Electrophysiol Clin 2016; 8 (2): 411-421.

26. Sardar MR, Saeed W, Kowey PR. Antiarrhythmic Drug Therapy for Atrial Fibrillation. Heart Failure Clin 2016; 12 (2): 205-221.

27. van Hunnik A, Lau DH, Zeemering S, Kuiper M, Verheule S, Schotten U. Antiarrhythmic effect of vernakalant in electrically remodeled goat atria is caused by slowing of conduction and prolongation of postrepolarization refractoriness. Heart Rhythm 2016; 13 (4): 964-972.

28. Christophersen IE, Olesen MS, Liang B, Andersen MN, Larsen AP, Nielsen JB et al. Genetic variation in KCNA5: impact on the atrialspecific potassium current IKur in patients with lone atrial fibrillation. Eur Heart J 2013; 34 (20): 1517-1525.

29. Ravens U, Wettwer E. Ultra-rapid delayed rectifier channels: molecular basis and therapeutic implications. Cardiovasc Res 2011; 89: 776-785.

30. Nattel S, Harada M. Atrial remodeling and atrial fibrillation: recent advances and translational perspectives. J Am Coll Cardiol 2014; (63): 2335-2345.

31. Clauss S, Sinner MF, Kääb S, Wakili R. The Role of MicroRNAs in Antiarrhythmic Therapy for Atrial Fibrillation. Arrhythm Electrophysiol Rev 2015; 4 (3): 146-155. DOI: 10.15420/aer.2015.4.3.146.

32. Bosch RF, Zeng X, Grammer JB, Popovic K, Mewis C, Kühlkamp V. Ionic mechanisms of electrical remodeling in human atrial fibrillation. Cardiovasc Res 1999; 44: 121-131.

33. Allessie MA, Boyden PA, Camm AJ, Kléber AG, Lab MJ, Legato MJ et al. Pathophysiology and prevention of atrial fibrillation. Circulation 2001; 103: 769-777.
34. Scherer D, Seyler C, Xynogalos P, Scholz EP, Thomas D, Backs J et al. Inhibition of Cardiac Kir Current (IK1) by Protein Kinase C Critically Depends on PKC $\beta$ and Kir2.2. PLoS One 2016; 11 (5): e0156181. DOI: 10.1371/journal.pone.0156181.

35. Workman AJ, Marshall GE, Rankin AC, Smith GL, Dempster J. Transient outward $\mathrm{K}+$ current reduction prolongs action potentials and promotes afterdepolarisations: a dynamic-clamp study in human and rabbit cardiac atrial myocytes. J Physiol 2012; 590 (17): 4289-4305.

36. Olesen MS, Refsgaard L, Holst AG, Larsen AP, Grubb S, Haunsø $\mathrm{S}$ et al. A novel KCND3 gain-of- function mutation associated with early-onset of persistent lone atrial fibrillation. Cardiovasc Res 2013; 98 (3): 488-495.

37. Li GR, Dong MQ. Pharmacology of cardiac potassium channels. Adv Pharmacol 2010; 59: 93-134.

38. Holmes AP, Yu TY, Tull S, Syeda F, Kuhlmann SM, O’Brien SM et al. A Regional Reduction in Ito and IKACh in the Murine Posterior Left Atrial Myocardium is Associated with Action Potential Prolongation and Increased Ectopic Activity. PLoS One 2016; 11 (5): e0154077. DOI: 10.1371/journal.pone.0154077.

39. Nielsen MW, Olesen MS, Refsgaard L, Haunsø S, Sven JH. Screening of the ito regulatory subunit klf15 in patients with early-onset lone atrial fibrillation. Front Genet 2013; 4 (88).

40. Dobrev D, Wettwer E, Kortner A, Knaut M, Schüler S, Ravens U. Human inward rectifier potassium channels in chronic and postoperative atrial fibrillation. Cardiovasc Res 2002; 54: 397.

41. Zhang C, Yuan GH, Cheng ZF, Xu MW, Hou LF, Wei FP. The single nucleotide polymorphisms of Kir3.4 gene and their correlation with lone paroxysmal atrial fibrillation in Chinese Han population. Heart Lung Circ 2009; 18 (4): 257-261.

42. Voigt N, Friedrich A, Bock M, Wettwer E, Christ T, Knaut M et al. Differential phosphorylation-dependent regulation of constitutively active and muscarinic receptor-activated IK,ACh channels in patients with chronic atrial fibrillation(J). Cardiovasc Res 2007; 74: 426-437.

43. Wang H, Li T, Zhang L, Yang Y, Zeng XR. Effects of intracellular calcium alteration on SK currents in atrial cardiomyocytes from patients with atrial fibrillation. Zhongguo Ying Yong Sheng Li Xue Za Zhi 2014; 30 (4): 296-300.

44. Ling TY, Wang XL, Chai Q, Lau TW, Koestler CM, Park SJ et al. Regulation of the SK3 channel by microRNA-499-potential role in atrial fibrillation. Heart Rhythm 2013; 10 (7): 1001-1009.

45. Diness JG, Bentzen BH, Sørensen US, Grunnet M. Role of Calciumactivated Potassium Channels in Atrial Fibrillation Pathophysiology and Therapy. J Cardiovasc Pharmacol 2015; 66 (5): 441-448. DOI: 10.1097/ FJC.0000000000000249.

46. Olesen MS, Jabbari J, Holst AG, Nielsen JB, Steinbrüchel DA, Jespersen $\mathbf{T}$ et al. Screening of KCNN3 in patients with early-onset lone atrial fibrillation. Europace 2011; 13 (7): 963-967.

Received September 24, 2020. Accepted February 2, 2021. 